\title{
Frustrated magnetic interactions in a cyclacene crystal
}

\author{
R. Ortiz $\odot,{ }^{1,2}$ J. C. Sancho-García $\odot,^{2}$ and J. Fernández-Rossier ${ }^{3, *}$ \\ ${ }^{1}$ Departamento de Física Aplicada, Universidad de Alicante, 03690 Sant Vicent del Raspeig, Spain \\ ${ }^{2}$ Departamento de Química Física, Universidad de Alicante, 03690 Sant Vicent del Raspeig, Spain \\ ${ }^{3}$ QuantaLab, International Iberian Nanotechnology Laboratory (INL), Av. Mestre José Veiga, 4715-330 Braga, Portugal
}

(Received 30 July 2021; accepted 8 December 2021; published 19 January 2022)

\begin{abstract}
We study the emergence of magnetism and its interplay with structural properties in a two-dimensional molecular crystal of cyclacenes, using density functional theory (DFT). Isolated cyclacenes with an even number of fused benzenes host two unpaired electrons in two topological protected zero modes, at the top and bottom carbon rings that form the molecule. We show that, in the gas phase, electron repulsion promotes an open-shell singlet with strong intramolecular antiferromagnetic exchange. We consider a closed packing triangular lattice crystal phase and we find a strong dependence of the band structure and magnetic interactions on the rotation angle of the cyclacenes with respect to the crystal lattice vectors. The orientational ground state maximizes the intermolecular hybridization, yet local moments survive. Intermolecular exchange is computed to be antiferromagnetic, and DFT predicts a broken symmetry $120^{\circ}$ spin phase reflecting the frustration of the intermolecular spin coupling. Thus, the cyclacene crystal realizes a bilayer of two antiferromagnetically coupled $S=1 / 2$ triangular lattices. Our results provide a bottom-up route towards carbon based strongly correlated platforms in two dimensions.
\end{abstract}

DOI: 10.1103/PhysRevMaterials.6.014406

\section{INTRODUCTION}

Magnetism and strongly correlated phases have been traditionally alien to the realm of graphitic crystals and polycyclic aromatic hydrocarbons (PAHs). Thus, graphite, graphene, and carbon nanotubes, when described in an independent electron approximation, feature wide bands with extended states (small or vanishing density of states at the Fermi energy) so that the effect of Coulomb interactions in these systems are mostly negligible. In spite of this, strong correlations and magnetism were predicted in graphene zigzag edges [1-3] and radical PAHs [4], but in both cases their strong reactivity is expected to compromise their chemical stability.

However, recent experimental breakthroughs have significantly changed this scenario. First, the discovery of strongly correlated electronic phases in twisted bilayer graphene $[5,6]$ has shown that narrow bands are indeed possible in suitably designed carbon based two-dimensional (2D) materials. Second, the on-surface assisted synthesis of multiradical PAHs, that remain stable in ultrahigh vacuum, and the study of their electronic excitations using scanning tunneling microcope spectrocopy (STS) have significantly paved the way towards the fine control of their synthesis. This includes triangulenes [7-10], extended triangulenes, double triangulenes [11] and rhombenes [12], among others $[9,13]$. Therefore, using STS [14] to probe the fluctuating local moments, from PAH with open-shell singlets $[9,10,13]$, is a firmly stablished tool for this kind of systems.

\footnotetext{
* On leave from Departamento de Física Aplicada, Universidad de Alicante, Spain.
}

An ingredient common to both twisted bilayer and radical PAHs is the presence of localized electronic states close to the Fermi energy that host the strongly correlated electrons. This leads us to propose here a bottom-up route to engineer strongly correlated carbon based 2D crystals. Our approach is to use as building blocks radical molecules that can assemble in such a way that intermolecular interactions lead to a weak hybridization of those molecular states hosting the unpaired electrons. In-plane assembly of radical PAHs leads, with some exceptions [9], to strong covalent bonding of the molecular states, compromising the ultimate goal of preserving the localized molecular levels.

Here we consider cyclacenes with diradical character as building blocks for a molecular crystal (see Fig. 1) with a weak intermolecular interaction, so that the diradical character can be preserved in the crystal phase. These aromatic hydrocarbon nanobelts [15] are very appealing molecules and are the subject of strong interest, both theoretically and experimentally. In the gas phase, the local moments are hosted by molecular orbitals that are topological symmetry protected zero modes [16]. A natural configuration for their self-assembly is a closed packing crystal, where the separation between molecules is in the range of the interlayer distance in graphite. We find this preserves the local moments of the isolated molecules in the crystal phase. Importantly, the resulting organization in this idealized cyclacene crystal yields a network of states that does not live in a bipartite lattice. This opens the door, as we will show next, to frustrated antiferromagnetic interactions that are known to promote quantum spin liquid states $[17,18]$, and thus different from the widely studied broken symmetry molecular magnetism [19] and from the Lieb theorem paradigm $[3,10,20,21]$. 

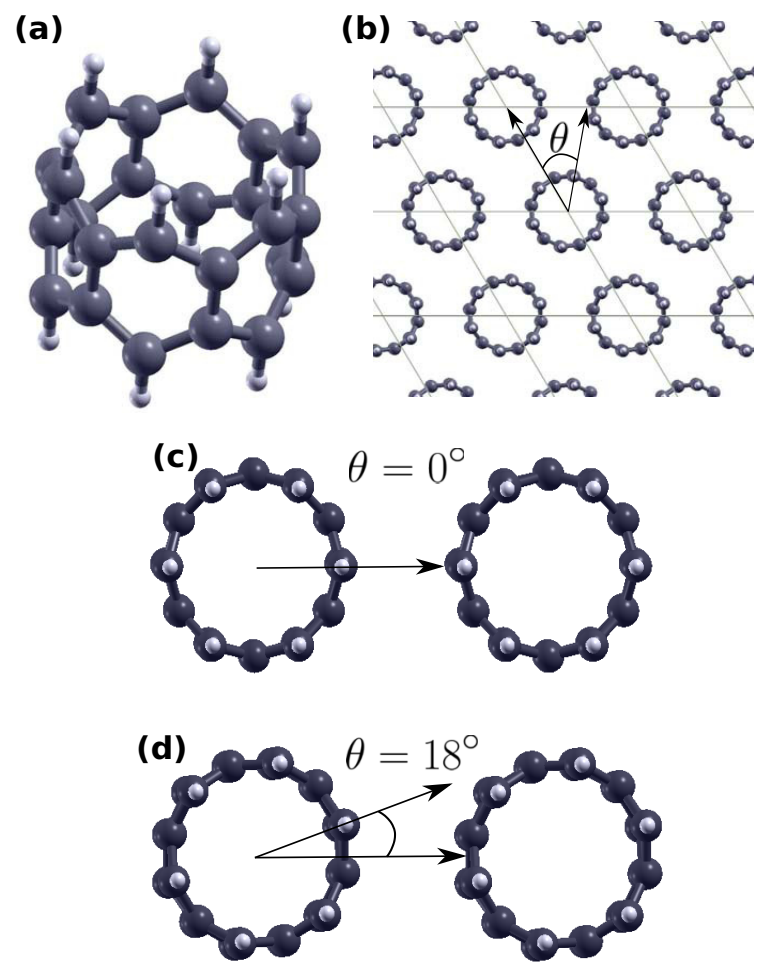

FIG. 1. (a) Chemical structure of [6]CC. (b) Molecular crystal lattice of [6]crystacene showing the rotational angle $\theta$. (c) and (d) display two cyclacenes with different relative angle $\theta$. Gray balls represent carbons and white ones represent hydrogens [22].

Cyclacenes, hereafter $[n] \mathrm{CC}$ 's, with $n$ denoting the number of fused benzene rings, can be thought of as the shortest possible carbon nanotubes with zigzag edges. The electronic properties of $[n] C C$ 's depend on the parity of $n$, showing an odd-even pattern [23-25]. This is confirmed at several levels of theory, where a simple one-orbital per atom tight-binding model yields two zero-energy states for [even]CC's and four states split into two pairs for [odd]CC's [26]. This provides a simple picture to understand the di/tetraradical character obtained by more sophisticated calculations [27]. On the experimental side, besides the multiple efforts to synthesize carbon nanobelts [28-34], the obtention of pristine cyclacenes is still missing.

In Fig. 1(a) we show the structure of [6]CC. We can think of this molecule as two carbon rings with a strong covalent coupling through the nonhydrogenated carbons (NHCs). Their tight-binding energy spectra presents two zero-energy states localized on the hydrogenated carbon (HC) sites. In this work, we consider a two-dimensional crystal of these [6]CC molecules, as if they had gone through a process of selfassembly over a surface [35-37] (hereafter [ $n$ ]crystacene [see Fig. 1(b)]. We assume that the molecules assemble in a triangular lattice, with the axis of the tubes perpendicular to the plane of the crystal. This idealized (monolayer) self-assembly agrees with that recently found for closely related systems, as cycloparaphenylene nanorings [38]. In order to characterize the orientational order of the crystal, we define the relative angle $\theta$ formed between the line joining the geometric center of the molecule and the projection of the hydrogenated carbon atoms on the plane containing the center. This angle is determined by minimization of the ground state energy obtained using DFT for different magnetic phases.

In the following we address the study of the electronic structure of the [6]crystacene, focusing on the emergence of molecular local moments, the nature of intermolecular exchange, and their interplay with the rotational angle $\theta$.

\section{METHODS}

We describe the electronic structure of cyclacenes with three different levels of theory. Individual molecules are described with a Hubbard model with a single orbital per carbon site. The model is treated both in the mean-field approximation [3] and using multiconfigurational methods in a complete active space (CAS) with six single-particle states and six electrons [14,21]. The molecular crystal is treated using density functional theory (DFT) calculations, carried out with the QUANTUM ESPRESSO (QE) code [39,40]. We use a PerdewBurke-Ernzerhof (PBE) [41] ultrasoft nonrelativistic density functional for both carbon and hydrogen atoms. The van der Waals (vdW) interactions, when considered, were implemented with the "vdw-DF" flag [42-44]. The kinetic energy cutoff considered for wave functions was $30 \mathrm{Ry}$, for the charge density and potential it was $700 \mathrm{Ry}$, and we employed a $k$-grid of $10 \times 10 \times 1$.

We consider five magnetic states for the crystal: a spinunpolarized nonmagnetic (NM) state, a ferromagnetic (FM) state, and three states with intramolecular antiferromagnetic correlations that differ by the intermolecular spin order: AF1, with ferromagnetic intermolecular correlations; AF2, a stripe phase with two antiferromagnetically coupled molecules per unit cell; and AF120, with noncollinear magnetic order where first neighbor spins are misaligned by $120^{\circ}$, and three molecules per unit cell [see Figs. 3(a)-3(e)].

In the case of NM, AF1, and AF2, we have explored the $\theta$ dependence of the ground state energy, using the atomic coordinates obtained upon relaxation of the unit cell at $\theta=0^{\circ}$ for these three magnetic configurations; this geometry was further employed for any angle $\theta$ and for the band structures. We have also computed the FM phase for two different angles $\theta=0^{\circ}$ and $\theta=18^{\circ}$ using the same relaxed coordinates as those employed for the AF120 phase, as we will explain in the following. The results for this ferromagnetic phase are in Table I.

In the case of the AF120 phase, the unit cell has three molecules. This makes the computational cost of the structural relaxation prohibitively large. We use instead atomic configurations obtained with smaller unit cells and we only consider $\theta=0^{\circ}$. We have tried two different structures and, in both cases, the magnetic ground state with three molecules in the unit cell converges to the 120 phase. Below we show the results obtained using the atomic coordinates obtained from the relaxation of the AF1 phase with $\theta=-20^{\circ}$, with the lattice constants $|\vec{a}|,|\vec{b}|=14.29 \AA$ (which corresponds to a center-to-center intermolecular distance of $8.25 \AA$ ): the approximate position of the energy minimum in the $E$ vs $|\vec{a}|,|\vec{b}|$ curve for the frustrated AF2 phase (see Supplemental Material [45]). No vdW interactions were considered for the AF120 phase. For the NM and AF2 phases we employed a 

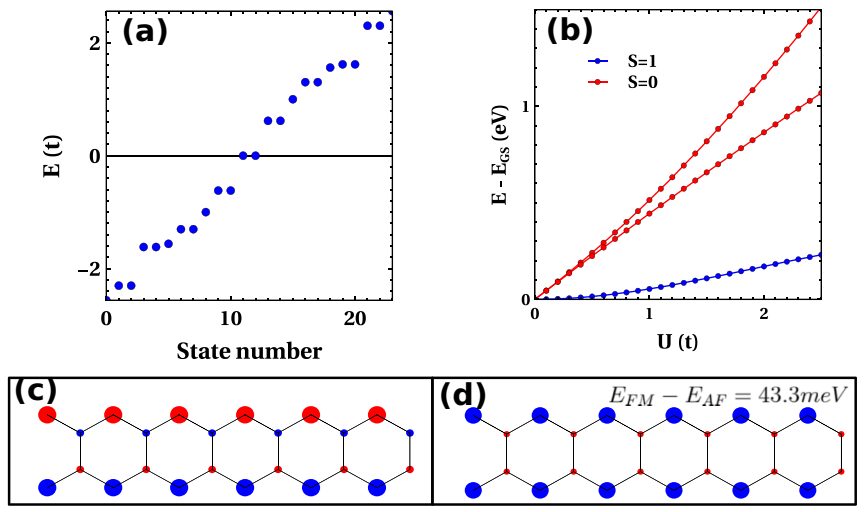

FIG. 2. Gas phase isolated molecule calculations. (a) Singleparticle tight-binding spectra for [6]CC, calculated for the planar lattices of panels (c) and (d) with periodic boundary conditions. Note that, as we mention in the text, finite-temperature DFT calculations [27] hold the diradical character of [even]CC, so ignoring the ring curvature on this matter is justified. (b) Excitation energies computed with the CAS $(6,6)$ approximation for the Hubbard model with $t=2.7 \mathrm{eV}$. The ground state has $S=0$. The $S=1$ state has $E_{S=1}=54 \mathrm{meV}$ for $U=t$. We take $t=2.7 \mathrm{eV}$, which has been widely used in the literature [47] and is very close to the value inferred from angle-resolved photoemission experiments in graphene [48]. (c),(d) Expectation value of spin density $S_{z}(i)$, calculated in the mean-field approximation of the Hubbard model with $U=t$ for two magnetic configurations, with total $S_{z}=0$ (AF) and total $S_{z}=1$ (FM). The AF is the ground state with $E_{\mathrm{FM}}-E_{\mathrm{AF}}=43.3 \mathrm{meV}$ for $U=t$ and $t=2.7 \mathrm{eV}$ in the mean field, not far from the CAS $(6,6)$ result.

Marzari-Vanderbilt smearing with a Gaussian spreading of $10^{-4} \mathrm{Ry}$, while no smearing at all was used for the AF1 or AF120 phases [46].

In order to characterize the magnetic state of the crystals, we use two indicators:

$$
M_{\text {ring }}^{\text {tot }}=\sum_{i \in \text { ring }} m_{i}
$$

and

$$
M_{\text {ring }}^{\mathrm{abs}}=\sum_{i \in \text { ring }}\left|m_{i}\right|,
$$

TABLE I. Energy and magnetization for different crystacene crystal phases. $\Delta E=E-E_{\mathrm{AF} 120}$ is the energy per cyclacene referred to the energy of the AF120 phase, in meV. The magnetic moments per ring are relative to those computed for the FM phase with $\theta=18^{\circ}$ [see Methods, Eq. (3)].

\begin{tabular}{lccl}
\hline \hline Configuration & $\Delta E(\mathrm{meV})$ & $\tilde{M}_{\text {ring }}^{\text {abs }}$ & $\tilde{M}_{\text {ring }}^{\text {tot }}$ \\
\hline AF120 & 0.0 & 0.89 & 0.68 \\
AF2 & 6.9 & 0.92 & 0.68 \\
NM & 44.6 & 0.0 & 0.0 \\
AF1 $\left(\theta=0^{\circ}\right)$ & 47.4 & 0.14 & 0.09 \\
AF1 $\left(\theta=18^{\circ}\right)$ & 105.8 & 1.08 & 0.82 \\
FM $\left(\theta=0^{\circ}\right)$ & 44.3 & 0.05 & 0.04 \\
FM $\left(\theta=18^{\circ}\right)$ & 409.2 & 1.0 & 1.0 \\
\hline \hline
\end{tabular}

(a)
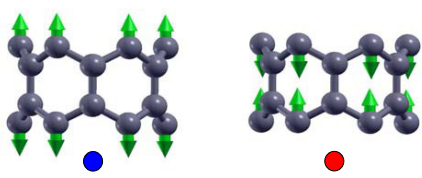

(b)
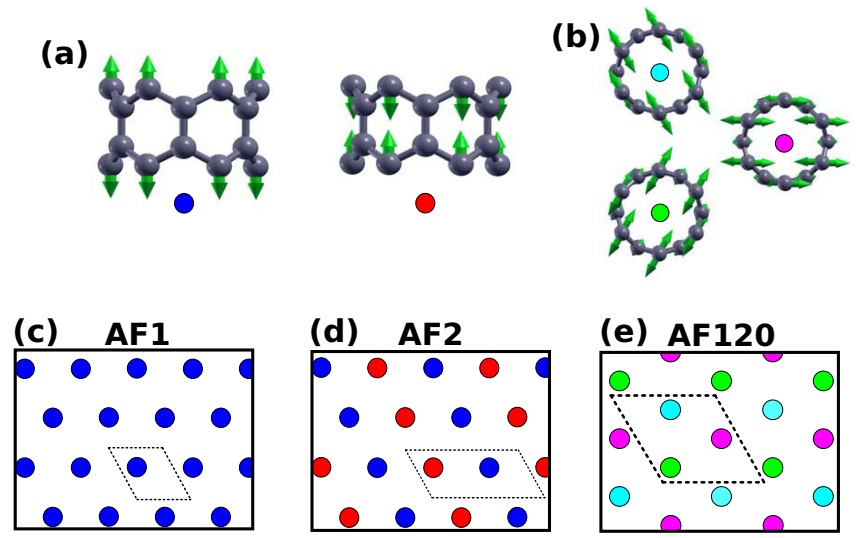

(e) AF 120
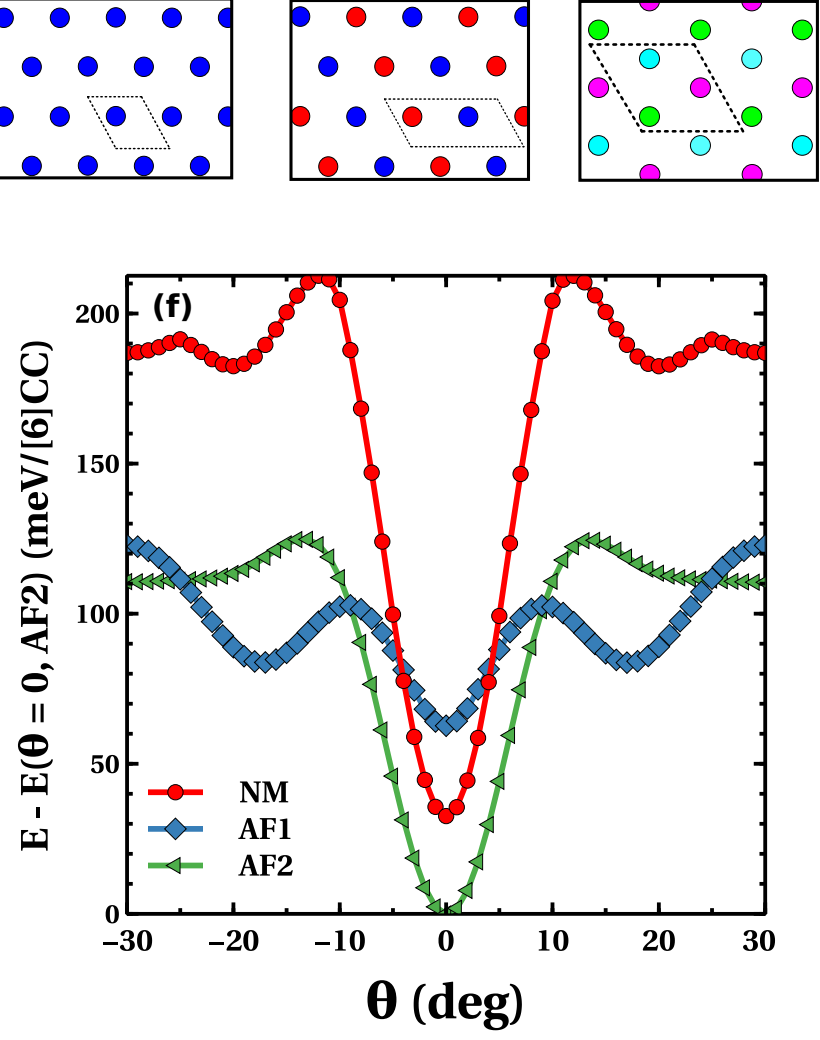

FIG. 3. (a) Calculated local moments of the AF2 collinear phase from a side perspective when $\theta=0^{\circ}$ and (b) AF120 phase. Hydrogens were omitted for clarity. The local moment per hydrogenated carbon is $\approx 0.04 \mu_{B}$ in both phases. (c), (d), and (e) are the schematic representations of the AF1, AF2, and AF120 phases (see text). (f) Ground state energy as a function of $\theta$, for the NM, AF1, and AF2 phases, referred to the value for the $\mathrm{AF} 2$ phase and $\theta=0^{\circ}$. For this calculation we employed the relaxed geometries and lattice vectors at $\theta=0^{\circ}$ for each magnetic configuration. Angle steps of $\Delta \theta=1^{\circ}$ were taken to calculate the curve shown.

where $m_{i}$ is the length of the atomic magnetic moments, as obtained from the output of our DFT calculations. The use of these two indicators is motivated by the fact that, in a given ring, there is a small residual magnetization in the nonhydrogenated carbon atoms, typical of the broken symmetry solutions in bipartite systems [3]. As a reference, the absolute magnetic moment per ring in the single-molecule mean-field calculation is $M_{\text {ring }}^{\text {abs }} \sim 1.6 \mu_{B}$ for the FM solution, to be compared with $M_{\text {ring }}^{\text {tot }}=1 \mu_{B}$.

The calculation of the atomic magnetic moments, carried out by QUANTUM ESPRESSO by integrating the magnetic density over a sphere centered around the atom, turns out to be nonreliable in this system. For instance, let us consider 
(a)
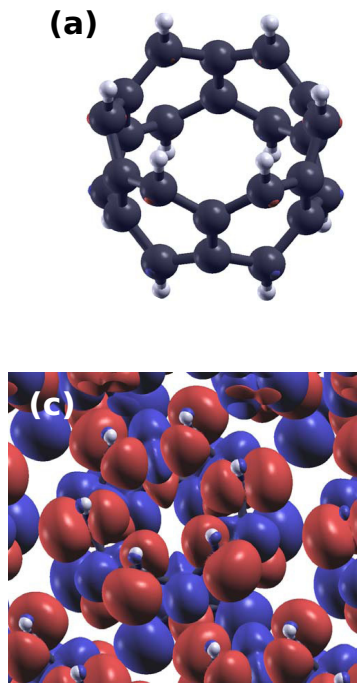

(b)
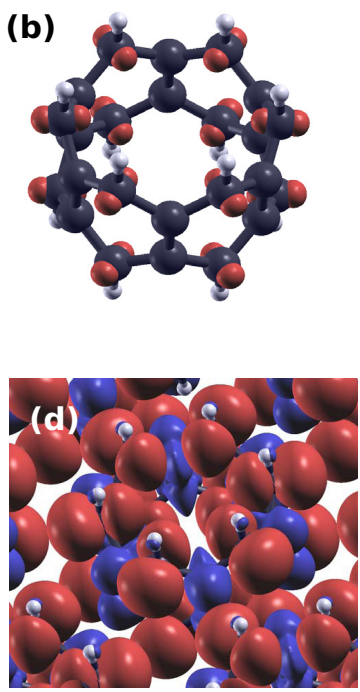

FIG. 4. Plot of the isosurfaces with the same isovalue [22] of the magnetic moments calculated with DFT for two different angles for the magnetic phases called in the text as (a),(c) AF1 with $\theta=0^{\circ}$ and $\theta=18^{\circ}$, respectively, and (b),(d) FM with $\theta=0^{\circ}$ and $\theta=18^{\circ}$, respectively. The color stands for the sign.

the FM phase in a strongly insulating case where the total moment per $\mathrm{CC}$ can be determined by counting the number of occupied bands per spin channel, and is unambiguously determined to be $2 \mu_{B}$. We can now obtain a lower limit for the magnetic moment per hydrogenated carbon atom using the following argument. The calculation of the magnetic density, shown in Fig. 4(d), shows that nonhydrogenated carbon atoms have a residual magnetization opposite to the dominant contribution of the hydrogenated carbon. We thus have $6\left(\left|m_{\mathrm{HC}}\right|-\left|m_{\mathrm{NHC}}\right|\right)=1 \mu_{B}$, the magnetization per ring. However, the value given by $\mathrm{QE}$ for this system is $6\left(\left|m_{\mathrm{HC}}\right|-\right.$ $\left.\left|m_{\mathrm{NHC}}\right|\right)=0.43 \mu_{B}$, which gives a good account of the unreliability of the integration method. We attribute the source of this discrepancy to the poor matching between a sphere and the $\pi$-shape electronic cloud that host the local moments (see Fig. 4).

For the phases with vanishing total spin per unit cell, we cannot rely on the use of the total moment to assess the error of the integration method. For this reason, in Table I we choose to normalize the values of the atomic moments obtained by QE for all phases to those obtained for the FM phase with $\theta=$ $18^{\circ}$, which is the angle with minimal intermolecular hopping. This local moment can be considered as an upper limit for the magnetization per ring. We thus define

$$
\tilde{M}_{\text {ring }}^{\text {tot }}=\frac{1}{M_{\text {ring }}^{\text {tot }}\left(\mathrm{FM}, \theta=18^{\circ}\right)} \sum_{i \in \text { ring }} m_{i}
$$

and analogously for the absolute magnetization.

\section{RESULTS AND DISCUSSION}

\section{A. Single CC}

Our starting point is the single-particle spectrum of the [6]CC molecule described with the tight-binding model with one orbital per atom. It features two zero modes whose

molecular orbitals are localized at the hydrogenated carbons of the top and bottom rings. These zero modes arise due to the combination of the chiral symmetry associated to the bipartite character of the [6]CC graph and the additional presence of the $C_{N}$ symmetry, when $N$ is even, in spite of the fact that the number of sites in both sublattices is the same.

The argument is similar to that presented by Koshino et al. [16]. We use the $C_{N}$ symmetry to represent the Hamiltonian of the molecule. This symmetry permits one to build the molecule as the repetition of $N$ blocks of four atoms. We label the eigenvalues of the $C_{N}$ symmetry as $\omega^{\ell}=e^{i k \ell}$, where $\ell=0, \ldots, N-1$. We thus have to impose $k=\frac{2 \pi}{N} m$, where $m=0,1, \ldots, N-1$, so that $\omega^{N}=1$. The Hamiltonian is thus block diagonal $\left[H=\sum_{k} H_{0}(k)\right]$, with

$$
H_{0}(k)=t\left(\begin{array}{cccc}
0 & 1+e^{i k} & 0 & 0 \\
1+e^{-i k} & 0 & 1 & 0 \\
0 & 1 & 0 & 1+e^{-i k} \\
0 & 0 & 1+e^{i k} & 0
\end{array}\right)
$$

where $t$ is the first neighbor hopping and the order of the basis goes from top to bottom in the unit cell. Now, for even $N$, we can have $k=\pi$ for $m=\frac{N}{2}$. This leads to $1+e^{i k}=$ $1+e^{-i k}=0$, so that the specific block has two zero modes, localized at the top and bottom rings. Effectively, the $k=\pi$ Hamiltonian is equivalent to the molecule with two disconnected zero modes, very much like the Clar's goblet [21]. Note that the argument can be trivially generalized to longer tubes.

Our next step is to study the effect of electron-electron interaction, within the Hubbard approximation. First we carry out a mean-field calculation for two configurations with $S_{z}=$ 0 (AF) and $S_{z}=1$ (FM). The expectation values of the local spin density $S_{z}(i)$ for the ground states of the AF and FM solutions are shown in Figs. 2(c) and 2(d) for $U=t$. The hydrogenated carbon atoms host a magnetic moment $g \mu_{B} S_{z}(i)$ close to $1 / 6$, the value expected for two unpaired electrons localized at the zero modes. The AF solution has an energy of $43.3 \mathrm{meV}$ smaller than the FM solution for $U=t$ and $t=2.7 \mathrm{eV}$.

The mean-field results are validated by the CAS $(6,6)$ calculations, with both exact diagonalization of the Hubbard model and more complex quantum chemistry calculations [49]. We systematically find that the ground state has $S=0$, complying with Lieb's theorem [20]. The first excited state has $S=1$, and the energy difference grows with $U$. For $U=t$ the energy difference is $53.8 \mathrm{meV}$, close to the mean-field value. The nature of the intramolecular antiferromagnetic interaction is Coulomb-driven exchange, very much like in bowtie diradicals $[13,21]$. The emerging picture is therefore that the ground state is an open-shell singlet, very much like the bowtie molecule, with two unpaired electrons hosted at the zero modes.

\section{B. Atomic structure of the crystal phases}

The combination of magnetic states, intermolecular distance, and angular orientation define a huge configurational space that can be explored only in part. For $\theta=0^{\circ}$ we find that the relaxed lattice constants for the NM phase were $|\vec{a}|,|\vec{b}| \approx$ $8.07 \AA$, for the AF1 phase $|\vec{a}|,|\vec{b}| \approx 8.18 \AA$, and for the AF2 
phase $|\vec{a}| \approx 16.25 \AA$ and $|\vec{b}| \approx 8.14 \AA$. The center-to-center distance $d$ can be related to the [6]CC radius by $d=2 R+\delta$, where $\delta \simeq 3.34 \AA$ for the AF2 phase, similar to the interlayer distance in graphite [50].

We find that, for the NM, AF1, and AF2 magnetic states, and reasonable intermolecular distances, the orientational ground state energy is minimized for $\theta=0^{\circ}$ [see Fig. 3(f)]. Figure 3(f) shows $E(\theta)$ for $\theta$ in a $60^{\circ}$ window, on account of the $C_{6}$ symmetry of the system. It is apparent that intermolecular exchange depends strongly on $\theta$. This figure shows that for $\theta=0^{\circ}, \mathrm{AF} 2$ has lower energy than NM and AF1 phases, and for these three magnetic states $\theta=0^{\circ}$ is the ground state.

Importantly, we find that AF2 has lower energy than NM for all values of $\theta$, showing that local moments survive in the crystal phase. In addition, we find that the lowest energy state for $\theta=0^{\circ}$ is the AF120 phase (Table I), but we could not carry out a $\theta$ dependence in this case. This type of noncollinear magnetic state is expected to arise as a broken spin symmetry solution in triangular lattices with antiferromagnetic exchange [51]. We therefore find that magnetic moments persist in the crystal phase and intermolecular exchange is antiferromagnetic. These are the central results of this work.

\section{Magnetization in the crystal phases}

Our next step is to address the key question of whether the open-shell nature of the molecules is preserved in the molecular crystal. This is assessed by computing the magnitude of the local moments per carbon ring that sheds light on whether the system remains in the open-shell strong-coupling limit of the single molecule, with local moments in line with those obtained in the gas phase. On the contrary, magnetic moments are quenched to some degree in the crystal phase.

As discussed in the methods part, it is convenient to refer our results to the case of the FM insulating crystal, for which the total magnetization is $2 \mu_{B}$ and the magnetic moment per carbon ring is $M_{\text {ring }}^{\text {tot }}=1 \mu_{B}$. From our calculations, summarized in Table I, we conclude that magnetic moments survive, to a large extent, in the crystal phase for the AF2 and AF120 phases at $\theta=0^{\circ}$ and for the AF1 phase at $\theta=18^{\circ}$, with small reductions, but they are severely quenched in the AF1 and FM phases for $\theta=0^{\circ}$. This reflects the strong interplay between orientational order and the survival of the magnetic moments. The energy differences presented in Table I are not expected to change qualitatively if different functionals are used. Specifically, the $120^{\circ}$ phase is expected to be the broken symmetry ground state in a triangular lattice with AF interactions. We note that the PBE functional has been previously used to model exchange interactions in planar nanographenes, with the comparison with experiments in fair agreement [52].

Inspection of Fig. 4 clearly shows that local moments are hosted by $\pi$ orbitals. A comparison of the diffferent panels shows how the magnitude of the atomic magnetic moments is very different in different crystal phases, in line with the results of Table I. It is apparent that moments are predominantly located on the hydrogenated carbon atoms, as anticipated in our single-molecule calculations with the Hubbard model. The nonhydrogenated carbons host moments opposite to the majority magnetization, as seen in planar nanographenes [3].
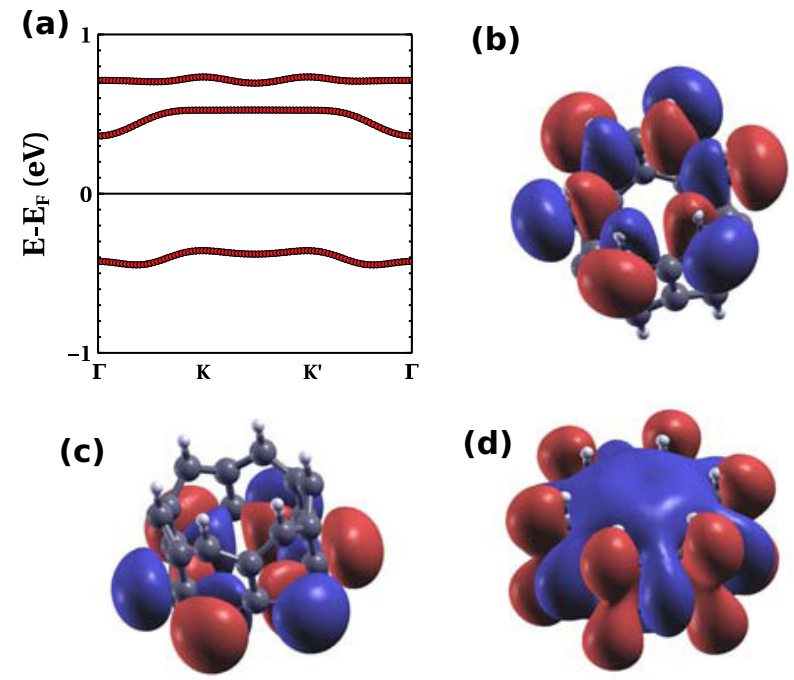

FIG. 5. (a) Band structure for AF1 phase and $\theta=18^{\circ}$. (b),(c),(d) WANNIER three-dimensional representations of the three bands in (a). Panels (b) and (c) corresponding to the bottom and middle bands match the zero-energy states from the single-molecule system. Panel (d) corresponds to the top band, which shows $\sigma$ character instead of $\pi$.

\section{Energy bands}

We now look for microscopic understanding of both the angular dependence of the ground state energy, as well as the differences between magnetic states. For that matter it is instructive to study the energy bands, as obtained from our DFT calculations. Naively, we expect that the bands closer to the Fermi energy are formed by the two zero modes of the diradical molecule. These states are a linear combination of $\pi$ orbitals localized along the radial direction of the molecules, which anticipate a strong dependence of the intermolecular hybridization on $\theta$.

Our energy band calculations for the AF1 phase for $\theta=$ $18^{\circ}$ [Fig. 5(a)] show that, in addition to the two relatively narrow bands arising from the in-gap zero modes of the molecule, there is a third band slightly above, that overlaps with the states above the Fermi energy. The angle $\theta=18^{\circ}$ corresponds to the second minima that appears in the curve of Fig. 3(f), which corresponds to a crystal geometry where carbon and hydrogen atoms avoid the alignment with those of their neighbors in order to minimize steric interactions. In this calculation we use the relaxed unit cell with $\theta=0^{\circ}$, and rigidly rotate the molecules to have $\theta=18^{\circ}$. A $k$ grid of $4 \times 4 \times 1$ was used instead since the convergence of this parameter is achieved earlier for insulators, and no smearing was used here either. These bands feature a twofold spin degeneracy that arises in centrosymmetric antiferromagnets.

In order to confirm the origin of these bands, we compute the Wannier wave functions using WANNIER90 [53-55]. We find three Wannier orbitals associated to the three narrow bands of Fig. 5(a). The ones corresponding to the first valence and conduction bands are localized in the top and bottom rings of the molecule, at the $\pi$ orbitals of the hydrogenated carbon atoms, as expected from our previous work for the molecular phase [26]. The one corresponding to the higher energy band 

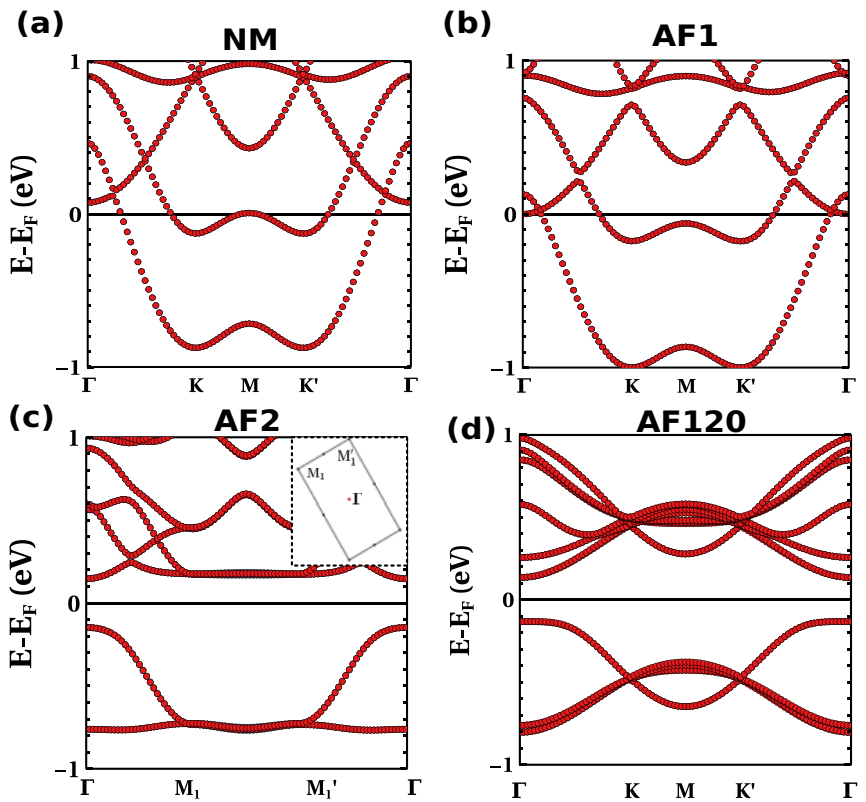

FIG. 6. Calculated band structure for the four magnetic configurations considered in [6]crystacene: (a) NM, (b) AF1, (c) AF2, and (d) AF120. $\theta=0^{\circ}$. The geometries are the same as those used for Fig. 3(f). Inset in (c) is the oblique lattice's first Brillouin zone with the high-symmetry points. For the collinear phases, the spin-up and spin-down bands are degenerate.

has a $\sigma$ character and a smaller bandwidth thereby, on account of the smaller intermolecular hopping.

The width of the energy bands depends strongly on $\theta$, as can be seen comparing Fig. 5(a) with Fig. 6(b), showing the bands of the AF1 phase for two different values of $\theta$. We see that the bandwidth $W$ of the valence band goes from less than $200 \mathrm{meV}$ for $\theta=18^{\circ}$ to more than $1.1 \mathrm{eV}$ for $\theta=0^{\circ}$. This dependence is a consequence of the highly directional nature of the $\pi$ orbitals that form these bands.

In turn, the modulation of the bandwidth has consequences on the local moment formation. The two energy scales that govern the emergence of local moments are the bandwidth $W$ of the bands at the Fermi energy and the effective Coulomb repulsion for double occupation of the molecular orbitals, that in the Hubbard aproximation is given by $\tilde{U}=U \sum_{i}|\psi(i)|^{4}$, where $\psi(i)$ is the amplitude of the molecular orbital at atom $i$ and $U$ is the atomic Hubbard parameter, which is in the range of $9 \mathrm{eV}$ [56]. Since $\psi(i)$ is equally distributed in six atoms, we estimate $\tilde{U}=U / 6 \simeq 1.5 \mathrm{eV}$. This is to be compared with the bandwidth.

In Figs. 6(a) and 6(b) we see how both the $\theta=0^{\circ} \mathrm{NM}$ and the AF1 phases are conducting and have a Fermi surface with two pockets. In contrast, the AF2 and AF120 phases [Figs. 6(c) and 6(d)], that are more stable in energy, are insulating, also for $\theta=0^{\circ}$. This shows the interplay between the intermolecular spin correlations and conduction.

Our results suggest that [6]crystacene provides a physical realization of two triangular monolayers that host local moments, with strong intralayer antiferromagnetic interactions, and antiferromagnetic interlayer exchange. In the AF120 phase the quenching of the local moments, relative to the gas phase, is small compared to the molecular case, so the system provides a physical realization of the triangular lattice bilayer Heisenberg model [57]. The nature of the ground state of the $S=1 / 2$ triangular monolayer has been studied for five decades now $[17,18,58,59]$. Using both quantum Monte Carlo and exact diagonalizations it was established that the ground state of the $S=1 / 2$ Heisenberg model with first neighbor AF coupling features long-range order with gapless Goldstone mode excitations and depleted magnetic moments, on account of enhanced quantum fluctuations [59]. For the triangular bilayer [57] it was found that, depending on the ratio $\lambda=\frac{J}{g}$ between intralayer $J$ and interlayer $g$ exchange, the spectrum is gapped for small $\lambda$, and gapless for $\lambda>1.4$, signaling the critical value for the transition between a quantum disordered state and a ground state with broken symmetry and long-range order. Interestingly, intramolecular exchange could be modified if we consider a crystal of longer cyclacenes. Therefore, this platform could permit one to study the quantum phase transition that may occur when the ratio of intramolecular and intermolecular exchange is modified.

As for the electronic properties of the broken symmetry AF120 phase predicted by our DFT results, we expect a vanishing Chern number [60] as well as a vanishing Berry curvature, and thereby null anomalous Hall effect. In contrast, at three-quarters filling the ground state of a triangular lattice is the so-called $Q$ phase predicted by Martin and Batista $[61,62]$, where the spins point to the corners of a tetrahedron, and there is a quantized anomalous Hall conductivity [61]. The study of this phase is out of the scope of the present paper.

\section{DISCUSSION AND CONCLUSIONS}

We have studied different magnetic configurations from a molecular crystal consisting of a two-dimensional triangular array of cyclacenes. In the gas phase, we have explored the diradical nature of the molecules using both multiconfigurational and mean-field calculations for the Hubbard model. We have shown that the diradical nature of the molecules has a topological origin, which leads to an open-shell $S=0$ singlet formed by two antiferomagnetically coupled electrons localized at the top and bottom rings of the molecule. We have studied the fate of these local moments in the crystal phase, using density functional theory. We have explored both the relative orientation of the molecules with respect to the crystal lattice vectors, characterized by the angle $\theta$, and we have considered four different magnetic states: NM and four types of magnetic order, FM, AF1, AF2, AF120. We find a strong dependence of the electronic structure with $\theta$. For all angles, the AF2 magnetic solution has lower energy than the NM case, showing that the antiferromagnetically correlated local moments of the gas phase survive in the crystal. We find that the ground state occurs for the AF120 phase, for $\theta=0^{\circ}$, which indicates antiferromagnetic intermolecular interactions.

In principle, the fate of these magnetic configurations might change due to interactions with the substrate. On the theory front, a previous work by two of us [63] shows that coupling to a conducting surface indeed renormalizes the 
spin excitation energies, but does not eliminate the openshell nature of the nanographenes. In addition, there is now a growing body of experimental work reporting magnetism on nanographenes deposited on gold $[8-13,64]$. We also note that it may be possible to find synthetic routes of the proposed system that lead to the formation of a cyclacene crystal on top of an insulator.

Therefore, our results portray the crystacene crystal as a versatile platform to promote strongly correlated phases, akin to the twisted bilayer graphene.

\section{ACKNOWLEDGMENTS}

We acknowledge J. L. Lado and A. Perez Guardiola for fruitful discussions. We acknowledge funding support from Ministry of Science and Innovation of Spain (Grants No. PID2019-106114GB-I00 and No. PID2019-109539GB), Generalitat Valenciana and Fondo Social Europeo (Grant No. ACIF/2018/175), Generalitat Valenciana (Grant No. Prometeo2017/139), and FEDER/Junta de Andalucía Consejería de Transformación Económica, Industria, Conocimiento y Universidades, Grant No. PY18-4834.
[1] K. Nakada, M. Fujita, G. Dresselhaus, and M. S. Dresselhaus, Phys. Rev. B 54, 17954 (1996).

[2] M. Fujita, K. Wakabayashi, K. Nakada, and K. Kusakabe, J. Phys. Soc. Jpn. 65, 1920 (1996).

[3] J. Fernández-Rossier and J. J. Palacios, Phys. Rev. Lett. 99, 177204 (2007).

[4] Y. Morita, S. Suzuki, K. Sato, and T. Takui, Nat. Chem. 3, 197 (2011).

[5] Y. Cao, V. Fatemi, A. Demir, S. Fang, S. L. Tomarken, J. Y. Luo, J. D. Sanchez-Yamagishi, K. Watanabe, T. Taniguchi, E. Kaxiras et al., Nature (London) 556, 80 (2018).

[6] Y. Cao, V. Fatemi, S. Fang, K. Watanabe, T. Taniguchi, E. Kaxiras, and P. Jarillo-Herrero, Nature (London) 556, 43 (2018).

[7] N. Pavliček, A. Mistry, Z. Majzik, N. Moll, G. Meyer, D. J. Fox, and L. Gross, Nat. Nanotechnol. 12, 308 (2017).

[8] S. Mishra, D. Beyer, K. Eimre, J. Liu, R. Berger, O. Groning, C. A. Pignedoli, K. Müllen, R. Fasel, X. Feng et al., J. Am. Chem. Soc. 141, 10621 (2019).

[9] S. Mishra, D. Beyer, K. Eimre, R. Ortiz, J. Fernández-Rossier, R. Berger, O. Gröning, C. A. Pignedoli, R. Fasel, X. Feng et al., Angew. Chem., Int. Ed. 59, 12041 (2020).

[10] S. Mishra, G. Catarina, F. Wu, R. Ortiz, D. Jacob, K. Eimre, J. Ma, C. A. Pignedoli, X. Feng, P. Ruffieux et al., Nature (London) 598, 287 (2021).

[11] J. Li, S. Sanz, J. Castro-Esteban, M. Vilas-Varela, N. Friedrich, T. Frederiksen, D. Peña, and J. I. Pascual, Phys. Rev. Lett. 124, 177201 (2020).

[12] S. Mishra, X. Yao, Q. Chen, K. Eimre, O. Gröning, R. Ortiz, M. Di Giovannantonio, J. C. Sancho-García, J. Fernández-Rossier, C. A. Pignedoli et al., Nat. Chem. 13, 581 (2021).

[13] S. Mishra, D. Beyer, K. Eimre, S. Kezilebieke, R. Berger, O. Gröning, C. A. Pignedoli, K. Müllen, P. Liljeroth, P. Ruffieux et al., Nat. Nanotechnol. 15, 22 (2020).

[14] R. Ortiz and J. Fernández-Rossier, Prog. Surf. Sci. 95, 100595 (2020).

[15] Q.-H. Guo, Y. Qiu, M.-X. Wang, and J. F. Stoddart, Nat. Chem. 13, 402 (2021).

[16] M. Koshino, T. Morimoto, and M. Sato, Phys. Rev. B 90, 115207 (2014).

[17] P. Anderson, Mater. Res. Bull. 8, 153 (1973).

[18] L. Balents, Nature (London) 464, 199 (2010).

[19] D. Gatteschi, R. Sessoli, and J. Villain, Molecular Nanomagnets (Oxford University Press on Demand, 2006), Vol. 5.
[20] E. H. Lieb, Phys. Rev. Lett. 62, 1201 (1989).

[21] R. Ortiz, R. Á. Boto, N. García-Martínez, J. C. Sancho-García, M. Melle-Franco, and J. Fernández-Rossier, Nano Lett. 19, 5991 (2019).

[22] A. Kokalj, Comput. Mater. Sci. 28, 155 (2003).

[23] H. S. Choi and K. S. Kim, Angew. Chem., Int. Ed. 38, 2256 (1999).

[24] Z. Chen, D.-e. Jiang, X. Lu, H. F. Bettinger, S. Dai, P. v. R. Schleyer, and K. N. Houk, Org. Lett. 9, 5449 (2007).

[25] C.-S. Wu, P.-Y. Lee, and J.-D. Chai, Sci. Rep. 6, 37249 (2016).

[26] A. Pérez-Guardiola, R. Ortiz-Cano, M. E. Sandoval-Salinas, J. Fernández-Rossier, D. Casanova, A. Pérez-Jiménez, and J.-C. Sancho-Garcia, Phys. Chem. Chem. Phys. 21, 2547 (2019).

[27] A. Pérez-Guardiola, M. E. Sandoval-Salinas, D. Casanova, E. San-Fabián, A. Pérez-Jiménez, and J.-C. Sancho-Garcia, Phys. Chem. Chem. Phys. 20, 7112 (2018).

[28] G. Povie, Y. Segawa, T. Nishihara, Y. Miyauchi, and K. Itami, Science 356, 172 (2017).

[29] G. Povie, Y. Segawa, T. Nishihara, Y. Miyauchi, and K. Itami, J. Am. Chem. Soc. 140, 10054 (2018).

[30] Y. Han, S. Dong, J. Shao, W. Fan, and C. Chi, Angew. Chem., Int. Ed. 60, 2658 (2021).

[31] F. Schulz, F. García, K. Kaiser, D. Pérez, E. Guitián, L. Gross, and D. Peña, Angew. Chem., Int. Ed. 58, 9038 (2019).

[32] Q. Zhang, Y.-E. Zhang, S. Tong, and M.-X. Wang, J. Am. Chem. Soc. 142, 1196 (2020).

[33] H. Shudo, M. Kuwayama, Y. Segawa, and K. Itami, Chem. Sci. 11, 6775 (2020).

[34] T.-H. Shi, Q.-H. Guo, S. Tong, and M.-X. Wang, J. Am. Chem. Soc. 142, 4576 (2020).

[35] S. Xu, S. J. Cruchon-Dupeyrat, J. C. Garno, G.-Y. Liu, G. Kane Jennings, T.-H. Yong, and P. E. Laibinis, J. Chem. Phys. 108, 5002 (1998).

[36] C. D. Bain and G. M. Whitesides, Science 240, 62 (1988).

[37] T. Fukui, S. Kawai, S. Fujinuma, Y. Matsushita, T. Yasuda, T. Sakurai, S. Seki, M. Takeuchi, and K. Sugiyasu, Nat. Chem. 9, 493 (2017).

[38] A. Pérez-Guardiola, Á. J. Pérez-Jiménez, L. Muccioli, and J. C. Sancho-García, Adv. Mater. Interfaces 6, 1801948 (2019).

[39] P. Giannozzi, S. Baroni, N. Bonini, M. Calandra, R. Car, C. Cavazzoni, D. Ceresoli, G. L. Chiarotti, M. Cococcioni, I. Dabo et al., J. Phys.: Condens. Matter 21, 395502 (2009).

[40] P. Giannozzi, O. Andreussi, T. Brumme, O. Bunau, M. B. Nardelli, M. Calandra, R. Car, C. Cavazzoni, D. Ceresoli, M. Cococcioni et al., J. Phys.: Condens. Matter 29, 465901 (2017). 
[41] J. P. Perdew, K. Burke, and M. Ernzerhof, Phys. Rev. Lett. 77, 3865 (1996).

[42] T. Thonhauser, S. Zuluaga, C. A. Arter, K. Berland, E. Schröder, and P. Hyldgaard, Phys. Rev. Lett. 115, 136402 (2015).

[43] T. Thonhauser, V. R. Cooper, S. Li, A. Puzder, P. Hyldgaard, and D. C. Langreth, Phys. Rev. B 76, 125112 (2007).

[44] K. Berland, V. R. Cooper, K. Lee, E. Schröder, T. Thonhauser, P. Hyldgaard, and B. I. Lundqvist, Rep. Prog. Phys. 78, 066501 (2015).

[45] See Supplemental Material at http://link.aps.org/supplemental/ 10.1103/PhysRevMaterials.6.014406 for the relaxed geometries of the different collinear magnetic phases and the energy profile as a function of the lattice vectors.

[46] We have verified that AF1 phase's energies do not depend on the smearing, which was introduced to improve the convergence of the calculations in the neighborhood of $\theta=0^{\circ}$.

[47] A. H. Castro Neto, F. Guinea, N. M. R. Peres, K. S. Novoselov, and A. K. Geim, Rev. Mod. Phys. 81, 109 (2009).

[48] A. Bostwick, T. Ohta, T. Seyller, K. Horn, and E. Rotenberg, Experimental determination of the spectral function of graphene, Lawrence Berkeley National Laboratory (2006).

[49] D. Sadowsky, K. McNeill, and C. J. Cramer, Faraday Discuss. 145, 507 (2010).

[50] G. Bacon, Acta Crystallogr. 4, 558 (1951).

[51] Y. Akagi and Y. Motome, J. Phys. Soc. Jpn. 79, 083711 (2010).
[52] T. Wang, S. Sanz, J. Castro-Esteban, J. Lawrence, A. Berdonces-Layunta, M. S. Mohammed, M. Vilas-Varela, M. Corso, D. Peña, T. Frederiksen et al., Nano Lett. 22, 164 (2022).

[53] A. A. Mostofi, J. R. Yates, G. Pizzi, Y.-S. Lee, I. Souza, D. Vanderbilt, and N. Marzari, Comput. Phys. Commun. 185, 2309 (2014).

[54] N. Marzari and D. Vanderbilt, Phys. Rev. B 56, 12847 (1997).

[55] I. Souza, N. Marzari, and D. Vanderbilt, Phys. Rev. B 65, 035109 (2001).

[56] T. O. Wehling, E. Şaşığlu, C. Friedrich, A. I. Lichtenstein, M. I. Katsnelson, and S. Blügel, Phys. Rev. Lett. 106, 236805 (2011).

[57] R. R. P. Singh and N. Elstner, Phys. Rev. Lett. 81, 4732 (1998).

[58] B. Bernu, P. Lecheminant, C. Lhuillier, and L. Pierre, Phys. Rev. B 50, 10048 (1994).

[59] L. Capriotti, A. E. Trumper, and S. Sorella, Phys. Rev. Lett. 82, 3899 (1999).

[60] H. Takatsu, S. Yonezawa, S. Fujimoto, and Y. Maeno, Phys. Rev. Lett. 105, 137201 (2010).

[61] I. Martin and C. D. Batista, Phys. Rev. Lett. 101, 156402 (2008).

[62] G.-W. Chern and C. D. Batista, Phys. Rev. Lett. 109, 156801 (2012).

[63] D. Jacob, R. Ortiz, and J. Fernández-Rossier, Phys. Rev. B 104, 075404 (2021).

[64] S. Mishra, D. Beyer, R. Berger, J. Liu, O. Gröning, J. I. Urgel, K. Müllen, P. Ruffieux, X. Feng, and R. Fasel, J. Am. Chem. Soc. 142, 1147 (2020). 